\title{
Assessment of forearm and plantar foot load in the elderly using a four-wheeled walker with armrest and the effect of armrest height
}

This article was published in the following Dove Press journal:

Clinical Interventions in Aging

14 October 2014

Number of times this article has been viewed

\section{Chang-Yong Ko* \\ Sol-Bi Kim* \\ Hyuk-Jae Choi \\ Yunhee Chang \\ Sungjae Kang \\ Yoon Heo \\ Jeicheong Ryu \\ Gyoosuk Kim \\ Museong Mun}

Korea Orthopedics and Rehabilitation

Research Center, Incheon, South

Korea

*These authors contributed equally to this work
Correspondence: Chang-Yong Ko Korea Orthopedics and Rehabilitation Research Center, 26, Gyeongin-ro 10 Beon-gil, Bupyeong-gu, Incheon, 403-I 20, South Korea

Tel +823 25095244

Fax +823 25I 29794

Email cyko@korec.re.kr
Background: Patients with hand and/or wrist pathology are recommended to have a four-wheeled walker with an arm rest (FWW-AR) rather than a standard walker or a standard four-wheeled walker (FWW). However, only a few quantitative studies have been performed to compare upper and lower extremity weight bearing. The aim of this study was to evaluate forearm and foot weight bearing using a FWW-AR and the effect of the armrest height.

Methods: Eleven elderly women (mean age $80.1 \pm 5.3$ years; mean height $148.5 \pm 4.0 \mathrm{~cm}$; mean weight $51.2 \pm 9.0 \mathrm{~kg}$ ) were enrolled. The subjects walked with an FWW-AR, with the elbow in either 90 degree (D90) or 130 degree (D130) flexion, for a distance of $10 \mathrm{~m}$. Surface electromyographic signals were recorded for the upper, middle, and lower trapezius, anterior deltoid, and erector spinae muscles; walking velocity was measured with the subjects weight bearing on their feet and forearms while walking. Simultaneously, the maximum plantar and forearm loads during walking with an FWW-AR were measured.

Results: The normalized foot plantar loads were lower at D90 than at D130, while the normalized forearm load was higher at D90 than at D130 (all $P<0.05$; left foot, $7.9 \pm 0.1 \mathrm{~N} / \mathrm{kg}$ versus $8.8 \pm 0.1 \mathrm{~N} / \mathrm{kg}$; right foot, $8.6 \pm 0.2 \mathrm{~N} / \mathrm{kg}$ versus. $9.6 \pm 0.1 \mathrm{~N} / \mathrm{kg}$; left forearm, $1.8 \pm 0.5 \mathrm{~N} / \mathrm{kg}$ versus $0.8 \pm 0.2 \mathrm{~N} / \mathrm{kg}$; and right forearm, $2.0 \pm 0.5 \mathrm{~N} / \mathrm{kg}$ versus $1.0 \pm 0.2 \mathrm{~N} / \mathrm{kg}$, respectively). The surface electromyographic activity of the muscles involved in shoulder elevation and the walking velocity were both lower with the elbow at D90 than at D130 (all $P<0.05$; left upper trapezius, $98.7 \% \pm 19.5 \%$ versus $132.6 \% \pm 16.9 \%$; right upper trapezius, $83.4 \% \pm 10.6 \%$ versus $108.1 \% \pm 10.5 \%$; left anterior deltoid, $94.1 \% \pm 12.8 \%$ versus $158.6 \% \pm 40.4 \%$; right anterior deltoid, $99.1 \% \pm 15.0 \%$ versus $151.9 \% \pm 19.4 \%$; and velocity, $0.6 \pm 0.1 \mathrm{~m} / \mathrm{sec}$ versus $0.7 \pm 0.1 \mathrm{~m} / \mathrm{sec}$, respectively).

Conclusion: Weight bearing on the lower extremities is significantly reduced when the upper extremities are supported during walking with an FWW-AR. Furthermore, the weight bearing profile is dependent on the armrest height.

Keywords: armrest height, forearm, four-wheeled walker with armrest, plantar foot, weight bearing

\section{Introduction}

Ambulatory assistive devices are used for improving mobility and balance and for reducing lower body load during weight bearing in the elderly and in patients with gait disorders, muscle weakness, and/or musculoskeletal pain. ${ }^{1-3}$ Selection of an appropriate assistive device (ie, stick, cane, crutch, or walker) is important to ensure adequate assistance and improve safety during walking. This selection depends on the patient's physical, physiological, and psychological abilities, including upper body strength, 
physical endurance, cognitive function, judgment, vision, vestibular function, and living environment. ${ }^{2}$ Van Hook et al suggested a criterion for selecting the appropriate assistive device based on the user's physical ability. A cane is usually recommended for those who are able to maintain balance or bear weight on one upper extremity; in others, a walker is the appropriate choice. ${ }^{2}$

Both a cane and walker expand the effective support base during the stationary stance position and walking, thereby improving postural stability and support., ${ }^{1,2}$ However, there are several differences among assistive devices. Although these devices can reduce the load on the lower extremity during weight bearing, the magnitude of reduction is much greater with a walker than a cane. ${ }^{1}$ Additionally, using a cane may not be effective for load reduction on the hip joint. ${ }^{1}$ Using a walker enlarges the base of support and reduces the challenge of balancing solely on a single leg as when walking with a cane. Furthermore, hand-reaction forces and moments are generated bilaterally, leading to a stabilizing effect. ${ }^{1,4}$ Therefore, a walker might offer greater postural stability than a cane. ${ }^{1}$ This suggests that a walker may be an appropriate selection for those with impaired postural stability and for those who will benefit from a reduced lower extremity load during weight bearing activities.

Walkers are classified into two main types, ie, a fixed walker without wheels (standard walker) and a wheeledwalker. While the fixed walker provides great stability, it requires execution of upper extremity movements for lifting and propelling, and is associated with higher energy consumption. ${ }^{5}$ Alternatively, the wheeled walker provides mobility with higher speed and less energy cost. ${ }^{6}$ A fourwheeled walker (FWW) increases stability during motion, such as making turns when walking, thereby reducing the number of stumbles and falls. ${ }^{7}$ In addition to the biomechanical aspects mentioned above, an FWW requires less training and the least effort for lifting and propelling compared with the cane and standard walker. ${ }^{7}$

When walking with an FWW, the individual's weight is supported by his/her wrist, resulting in excessive loads on the wrist. ${ }^{8}$ To propel an FWW, the user has to grip the FWW's handgrip. In addition, the user maintains a stooped position when walking with an FWW. Therefore, an FWW may be uncomfortable to use and may be inappropriate for those with back, hand, and wrist concerns. Alternatively, an FWW with an arm rest (FWW-AR) is recommended in these cases. ${ }^{2}$ Although biomechanical features, such as musculoskeletal load and weight bearing using walkers, have been evaluated in several studies, ${ }^{8-12}$ to the best of our knowledge, no studies have been conducted on the biomechanical assessment of walking with an FWW-AR. Therefore, the aim of this study was to investigate forearm and foot weight bearing, muscle activity of upper extremities, and walking velocity when using an FWW-AR. In addition, the effect of the armrest height was evaluated because the height of the FWW's handgrip constitutes an important criterion for effective use.

\section{Materials and methods Subjects}

A convenience sample of eleven elderly women (aged $80.1 \pm 5.3$ years) was recruited from the Welfare Center of the Elderly. Clinical measurements using a visual analog scale assessment, Mini-Mental State Examination-Korea test, and Berg Balance Scale test were performed to determine whether the subjects met the inclusion or exclusion criteria. The inclusion criteria were as follows: ability to walk a minimum of $10 \mathrm{~m}$ without an assistive device or other assistance, absence of central nervous system disorders, and absence of orthopedic and peripheral neurological disorders affecting the lower extremities. The subjects were excluded from the study sample if they met any of the following exclusion criteria: visual analog scale pain score in the extremities, including the waist $>3$ points, Mini-Mental Status Examination-Korea score $<24$ points, ${ }^{13}$ and Berg Balance Scale score $<45$ points. ${ }^{14}$ The subject demographic and anthropometric data are presented in Table 1. All the procedures were performed according to the protocol approved by the institutional review board of the Korea Orthopedics and Rehabilitation Engineering Center.

\section{Experimental procedures}

The subjects wore converse shoes and practiced walking using an FWW-AR for 10 minutes (KA-390; Paramount Bed Co Ltd., Tokyo, Japan) before the experiments. Afterwards, they walked with an FWW-AR at a self-selected walking speed along a $10 \mathrm{~m}$ walkway. The armrest height

Table I Demographic and anthropometric data of participants $(n=I I)$

\begin{tabular}{ll}
\hline General characteristics & Participants \\
\hline Age (years) & $80.1 \pm 5.3^{\mathrm{a}}$ \\
Height $(\mathrm{cm})$ & $148.5 \pm 4.0$ \\
Weight $(\mathrm{kg})$ & $51.2 \pm 9.0$ \\
BBS & $5 \mathrm{I} \pm 1.7$ \\
MMSE-K & $27.8 \pm 1.0$ \\
\hline
\end{tabular}

Note: aMean \pm standard deviation.

Abbreviations: BBS, Berg Balance Scale; MMSE-K, Mini-Mental State ExaminationKorea. 
was adjusted corresponding to 130 degrees (D130) of elbow flexion, which was the most comfortable angle for the participants in this study, or 90 degrees (D90) of elbow flexion (Figure 1). Kinematic and kinetic analyses were performed. The subjects were instructed to place their forearms on the armrest of the FWW-AR and to maintain an upright position during the walk.

Each subject initially performed three practice trials; the test was repeated five times at each elbow flexion angle. Data collection was initiated at the onset of the stance phase of the right foot and continued for ten steps with each leg. ${ }^{15}$ The maximum load was acquired for each of the five tests and then averaged. The subjects had a rest break of 10 minutes after the test session.

\section{Experimental devices \\ Surface electromyography}

Surface electromyography (sEMG) data were acquired simultaneously from the patient's bilateral upper, middle, and lower trapezius, anterior deltoid, and erector spinae muscles at the level of the third lumbar vertebra using a wireless surface EMG system (Trigno wireless system; Delsys, Boston, MA, USA) during walking with an FWW-AR. These muscles were selected to better reflect upper body motion and predict shoulder and neck pain when walking with an FWW-AR. Electrodes were placed using SENIAM (Surface Electromyography for Noninvasive Assessment of Muscles) recommendations. ${ }^{16}$ The raw sEMG was filtered by a band
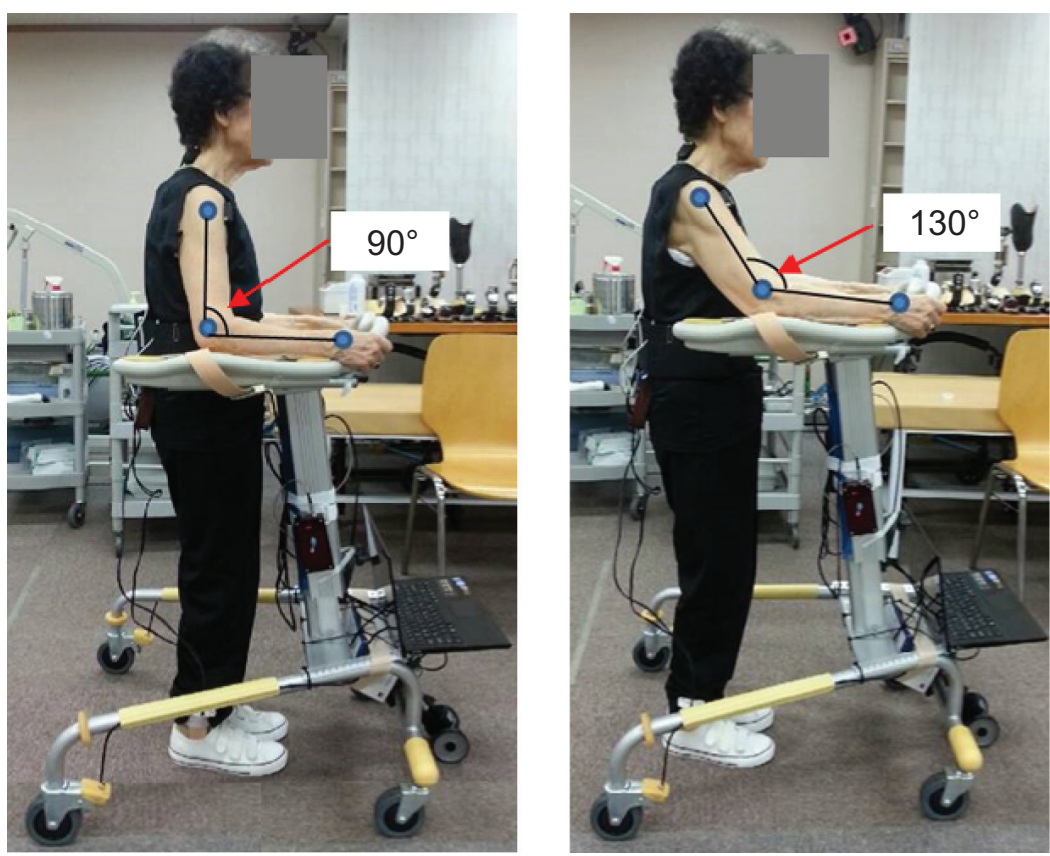

Figure I Experimental position of the elbow joint.

pass of $20-450 \mathrm{~Hz}$ and a common mode rejection ratio of $80 \mathrm{~dB}$. Data acquisition and processing was controlled using EMG Works software (Delsys). Data were collected at a sampling rate of $2,000 \mathrm{~Hz}$ and processed at 0.125 second intervals using the root mean square (RMS) method. ${ }^{17}$ The RMS values were normalized to the reference voluntary contraction (RVC), ${ }^{18}$ which was obtained during the walk without the FWW-AR and assistance. Maximum normalized RVC values were acquired for each of the five tests and then averaged.

\section{Plantar and forearm load measurements}

The maximum plantar and forearm loads during walking with an FWW-AR were measured using the Pedar in-shoe plantar pressure measurement system (Novel GmbH, Munich, Germany) at a sampling rate of $100 \mathrm{~Hz}$. In this study, two pairs of sensors were used: plantar pressure sensors attached on a pair of insoles and forearm pressure sensors attached on the armrest of the walker. Each subject was provided with appropriately sized converse shoes and a pair of Pedar insoles. Plantar and forearm pressure data were converted from Novel's proprietary format to ASCII format using Pedar-X Expert 12 software (Novel GmbH). The maximum force was then calculated and normalized to the subject's body weight.

\section{Walking velocity}

A wheeled incremental rotary encoder (ENC-1-1-24; Autonics, Busan, Republic of Korea) was attached on the FWW-AR 
and was in contact with the ground throughout the walk using the FWW-AR (Figure 1). Gait velocity was measured during walking with the FWW-AR.

\section{Statistical analysis}

The data were analyzed using Statistical Package for the Social Sciences version 20.0 software (IBM Corporation, Armonk, NY, USA). The Kolmogorov-Smirnov test was used to test the normality of distribution. sEMG activity, maximum forearm load, and velocity were analyzed using the paired $t$-test; maximum plantar load was compared using repeated-measures analysis of variance. Statistical significance was defined as $P<0.05$. The results of our analyses were expressed as the mean \pm standard error of the mean.

\section{Results}

The normalized maximum forearm and plantar loads are depicted in Figure 2 and Table 2. The normalized maximum plantar load showed a significant bilateral decrease during walking with an FWW-AR regardless of the forearm support height when compared with walking without an FWW-AR $(P<0.05)$. However, the load was lower during walking with the elbow at D90 compared with D130 $(P<0.05)$. In contrast with the results for the normalized maximum plantar load, the maximum forearm load was significantly higher during walking with the elbow at D90 than at D130 $(P<0.05)$.

sEMG recordings of the shoulder-neck muscles are shown in Figure 3 and Table 2. Significant decreases in left upper trapezius, left anterior deltoid, right upper and middle trapezius, and right anterior deltoid were observed with the elbow at D90 when compared with D130 $(P<0.05)$, whereas no differences were observed in the other muscles $(P>0.05)$. There was no significant difference in the normalized sEMG of erector spinae between D90 (left, $58.3 \% \pm 8.0 \%$; right, $74.8 \% \pm 7.5 \%$ ) and D130 (left, $53.9 \% \pm 8.3 \%$; right,

A

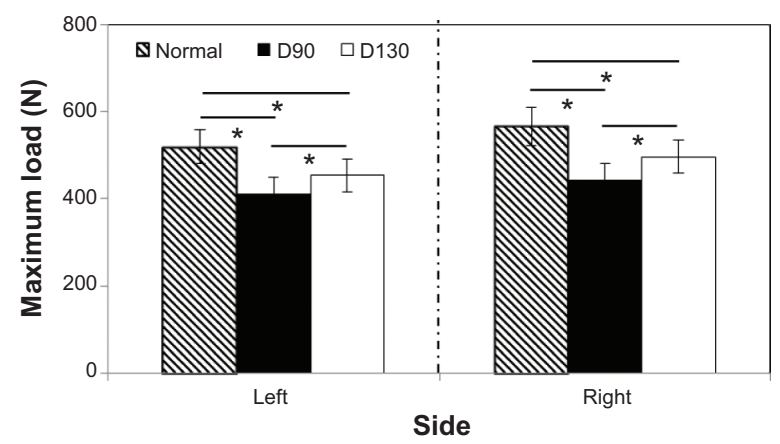

$78.6 \% \pm 8.1 \% ; P>0.05)$. The velocity during walking using the FWW-AR with the elbow at D90 $(0.6 \pm 0.1 \mathrm{~m} / \mathrm{sec})$ was significantly lower than with the elbow at D130 $(0.7 \pm 0.1 \mathrm{~m} / \mathrm{sec}$; $P<0.05)$.

\section{Discussion}

FWWs have been used widely as ambulatory-assistive devices for the elderly and for patients with gait disorders, muscle weakness, and/or musculoskeletal pain. However, FWWs might not be suitable for those with wrist, hand, or back problems due to the associated lifting mechanism and propelling involved, such as using the handgrip, excessive load on the wrist or hand, and a stooped posture. Therefore, FWW-ARs have been recommended for these individuals. According to many studies, correct use of an FWW is required to reduce the risk of falls and to maximize its function. The handgrip height is one of the most critical criteria for the correct use of the FWW. However, no studies have been conducted regarding the criteria for the FWW-AR, particularly for its armrest height. In this study, we investigated the load distribution on the forearm and the foot during walking with an FWW-AR and evaluated the effect of armrest height.

The maximum plantar loads were significantly lower, regardless of the height of the armrest, during walking with an FWW-AR than without an FWW-AR. Furthermore, the plantar load was lower with the elbow at D90 than at D130. However, the maximum forearm loads were significantly higher with the elbow at D90 than at D130. These complementary relationships between plantar and forearm loads implied that weight bearing on the plantar foot was reduced by the subjects' weight being supported by their forearm. Therefore, using an FWW-AR can reduce weight bearing on the lower extremity joints (ie, knee, ankle, or/and hip joints) by allowing partial support of body weight by the upper extremities. ${ }^{1,9}$

B

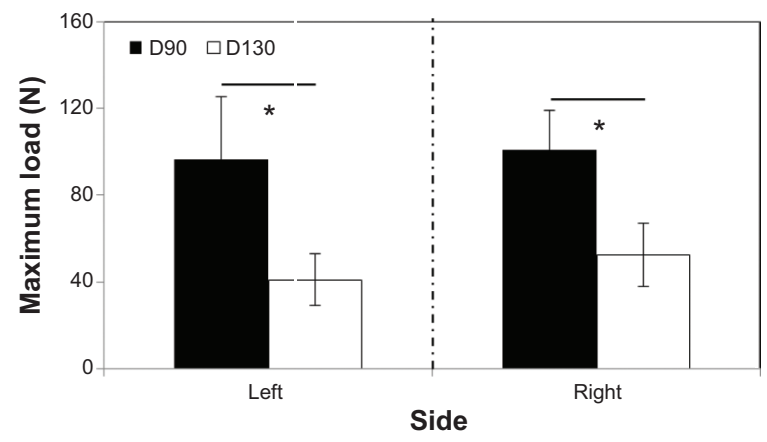

Figure 2 Results of maximum load (A) on the foot and (B) on the forearm. Notes: $* P<0.05$. D90, degrees of elbow flexion; DI30, 130 degrees of elbow flexion; Normal, without FWW-AR. 
Table 2 Results of normalized maximum load and normalized sEMG

\begin{tabular}{|c|c|c|c|c|c|c|c|}
\hline & & \multicolumn{3}{|l|}{ Left } & \multicolumn{3}{|l|}{ Right } \\
\hline & & D90 & DI30 & Normal & D90 & DI30 & Normal \\
\hline \multirow[t]{2}{*}{ Normalized maximum load (N/kg) } & Foot & $7.9 \pm 0.1^{\mathrm{a}}$ & $8.8 \pm 0.1^{*}$ & $10.1 \pm 0.0 * * *$ & $8.6 \pm 0.2$ & $9.6 \pm 0.1 *$ & $11.0 \pm 0.1 * * *$ \\
\hline & Forearm & $\mathrm{I} .8 \pm 0.5$ & $0.8 \pm 0.2 *$ & N/A & $2.0 \pm 0.5$ & $1.0 \pm 0.2^{*}$ & $\mathrm{~N} / \mathrm{A}$ \\
\hline \multirow[t]{5}{*}{ Normalized sEMG (\%) } & Upper trapezius & $98.7 \pm 19.5$ & $132.6 \pm 16.9 *$ & N/A & $83.4 \pm 10.6$ & $108.1 \pm 10.5 *$ & $\mathrm{~N} / \mathrm{A}$ \\
\hline & Middle trapezius & $78.4 \pm 8.4$ & $86.0 \pm 12.6$ & $\mathrm{~N} / \mathrm{A}$ & $74.8 \pm 13.1$ & $98.2 \pm 19.6^{*}$ & $\mathrm{~N} / \mathrm{A}$ \\
\hline & Lower trapezius & $86.2 \pm 13.1$ & $90.7 \pm 13.4$ & $\mathrm{~N} / \mathrm{A}$ & $95.7 \pm 19.7$ & $106.1 \pm 23.2$ & $\mathrm{~N} / \mathrm{A}$ \\
\hline & Anterior deltoid & $94.1 \pm 12.8$ & $158.6 \pm 40.4^{*}$ & $\mathrm{~N} / \mathrm{A}$ & $99.1 \pm 15.0$ & $151.9 \pm 19.4 *$ & $\mathrm{~N} / \mathrm{A}$ \\
\hline & Erector spinae & $58.3 \pm 8.0$ & $53.9 \pm 8.3$ & $\mathrm{~N} / \mathrm{A}$ & $74.8 \pm 7.5$ & $78.6 \pm 8.1$ & $\mathrm{~N} / \mathrm{A}$ \\
\hline
\end{tabular}

Notes: average \pm standard error; $* P<0.05$ (vs D90), ${ }^{* *} P<0.05$ (vs DI30).

Abbreviation: sEMG, surface electromyography.

The forearm load was higher during walking with an FWW-AR with the elbow at D90 than at D130, leading to an increase in shoulder joint forces. Excessive weight bearing load may negatively affect the integrity of the glenohumeral joint. ${ }^{19}$ Furthermore, the repetitive excessive net joint force may cause degeneration of the shoulder joint. ${ }^{20}$ Several researchers measured the vertical force exerted on a walker and then estimated the load on the glenohumeral joint. ${ }^{11,12}$ Melis et al measured the vertical forces exerted on a standard walker and a crutch in a patient with incomplete spinal cord injury and showed similar loads on the shoulder. Furthermore, they showed that the peak load was up to $100 \%$ of body weight and an average of $39 \%$ of body weight was placed on a standard walker when walking. ${ }^{11}$ Haubert et al compared the three-dimensional glenohumeral joint reaction force exerted on a front-wheeled walker and a crutch during walking in a patient with incomplete spinal cord injury. In contrast with the results of Haubert et al walking with a crutch caused significantly higher shoulder force than a walker. ${ }^{12}$ Therefore, a walker caused a remarkable load on the shoulder joint as shown in previous studies, which is consistent with the findings of the present study. Therefore, using an FWW-AR with the elbow at D90 might allow a higher load

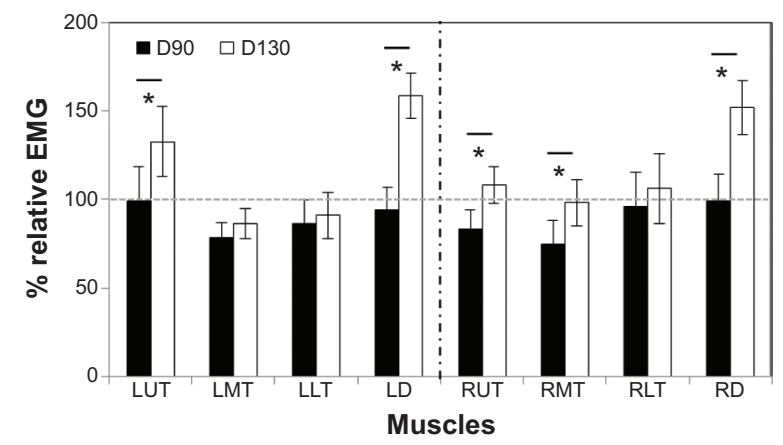

Figure 3 Results of normalized surface electromyography.

Notes: $* P<0.05$. D90, degrees of elbow flexion; DI30, 130 degrees of elbow flexion.

Abbreviations: EMG, electromyography; L, left; R, right; UT, upper trapezius; MT, middle trapezius; LT, lower trapezius; $D$, anterior deltoid. on the shoulder when compared with 130 degrees of elbow flexion, suggesting that D90 might be inadequate for the elderly with shoulder problems.

Higher sEMG activity was observed in the upper trapezius and deltoid muscles with the elbow at D130 than at D90 because of a higher elevation of the upper extremity at D130 compared with D90. Therefore, this result might be caused by the location of the armrest height during walking with an FWW-AR. It is well accepted that the trapezius sEMG activity can be used to estimate total shoulder neck muscle load; and unfavorable shoulder neck muscle load is one of the major risk factors of musculoskeletal disorders. ${ }^{21}$ Therefore, this issue during walking with an FWW-AR with the elbow at D130 would be important from the subject's point of view. In contrast with the differences in sEMG activity of the upper trapezius and deltoid muscle, there were no differences in the erector spinae sEMG between D90 and D130, suggesting that the armrest height has a lesser effect on torso kinematics. Together, the armrest height can affect shoulder-neck muscles.

The walking velocity with the FWW-AR was greater at D130 than at D90; however, the reason for this difference is unclear. However, this result might be explained by differences in the FWW-AR propulsion between walking at D90 and at D130. During walking using the FWW-AR with the elbow at D130, the upper extremity pressed the armrest of the FWW-AR diagonally. Therefore, there was a horizontal force component, which can augment the propulsion of the FWW-AR. However, the upper extremity pressed the armrest of the FWW-AR vertically at D90 and there was no horizontal force to propel the FWW-AR. Therefore, the propulsion may be relatively smaller during walking with the elbow at D130, resulting in an increase in velocity with the elbow at D130. Further studies to validate this hypothesis are needed. In addition to biomechanical aspects, emotional status can also affect human behavior, ${ }^{22-25}$ the velocity might be affected by avoidance behavior (ie, a negative emotion) or approach behavior (ie, a positive emotion). In this study, all subjects were more comfortable walking with 
the elbow at D130 than at D90, which may explain the increase in velocity with the elbow at D130.

This study had some limitations. All subjects were potential and not true FWW-AR users. Differences in gait, when walking with an FWW, between true and potential users have been reported previously. ${ }^{26,27}$ Furthermore, the subjects walked with an FWW-AR at a self-selected walking speed instead of a constant walking speed. Therefore, future studies involving true FWW-AR users with constant walking speeds are needed. We did not perform kinematic and kinetic analyses of the joint angle and moment and muscle force in the upper and lower body. Further studies should be conducted to investigate kinematic and kinetic features in the upper body, particularly the shoulder, and lower body to further understand the weight bearing distributions. In this study, we evaluated the muscle activity of the elevators (abductors), but not the shoulder adductors. Further studies to verify muscle activation and coactivation between abductors and abductors are necessary. Additionally, it has been previously shown that kinematic features in the elderly using the FWW are dependent on the subject's balance and the handgrip height of the FWW. ${ }^{28}$ Therefore, the balance and gait abilities of the elderly population and the subject's strategy for using the FWW-AR should be considered in future studies. We also did not consider differences in the distance between the subject and their FWW-AR, which might contribute to gait features due to stumbling or kicking the ambulatory assistive devices, and future studies should address this issue. Our tests were performed under indoor laboratory conditions with an even terrain. Therefore, it may be inappropriate to directly apply our findings to clinical/practical usages. Further studies are needed to verify clinical/practical usages, indoor versus outdoor use, and short-term use during rehabilitation versus a long-term solution for mobility/balance impairments.

\section{Conclusion}

Weight bearing on the lower extremity when walking with an FWW-AR, with the upper extremities supported, was significantly reduced regardless of the armrest height, but the magnitude of reduction was dependent on the armrest height. In addition to the differences noted in weight bearing on the upper and lower extremities, the sEMG activity of the shoulder and arm elevators and the walking velocities were different. Overall, our results suggest that when walking with an FWW-AR, having the elbow at D90 might be more suitable for reducing the lower extremity load during weight bearing than for mobility, whereas walking with the elbow at D130 might be more suitable for mobility.

\section{Acknowledgment}

This work was supported by the Quality of Life Technology Development Program (10047894, Development of indoor mobile device for elderly and disabled) funded by the Ministry of Trade, Industry and Energy (MOTIE, Korea).

\section{Author contributions}

All authors made substantial contributions to conception and design, and interpretation of data. All authors, except for JR, GK, and MM, collected the data. All authors participated in drafting the article or revising it critically for important intellectual content. All authors approved the final manuscript.

\section{Disclosure}

The authors report no conflicts of interest in this work.

\section{References}

1. Bateni H, Maki BE. Assistive devices for balance and mobility: benefits, demands, and adverse consequences. Arch Phys Med Rehabil. 2005; 86(1):134-145.

2. Van Hook FW, Demonbreun D, Weiss BD. Ambulatory devices for chronic gait disorders in the elderly. Am Fam Physician. 2003;67(8): 1717-1724.

3. Yeh HC. Elderly People's Use of and Attitudes Towards Assistive Devices. Brisbane, Australia: Queensland University of Technology; 2009.

4. Pardo RD, Deathe AB, Winter DA. Walker user risk index. A method for quantifying stability in walker users. Am J Phys Med Rehabil. 1993;72 (5):301-305.

5. Cetin E, Muzembo J, Pardessus V, Puisieux F, Thevenon A. Impact of different types of walking aids on the physiological energy cost during gait for elderly individuals with several pathologies and dependent on a technical aid for walking. Ann Phys Rehabil Med. 2010;53(6):399-405.

6. Holder CG, Haskvitz EM, Weltman A. The effects of assistive devices on the oxygen cost, cardiovascular stress, and perception of nonweightbearing ambulation. J Orthop Sports Phys Ther. 1993;18(4):537-542.

7. Kloos AD, Kegelmeyer DA, White SE, Kostyk SK. The impact of different types of assistive devices on gait measures and safety in Huntington's disease. PLoS One. 2012;7(2):e30903.

8. Mcquade KJ, Finley M, Oliveira AS. Upper extremity joint stresses during walkerassisted ambulation in post-surgical patients. Braz J Phys Ther. 2011;15(4):332-337.

9. Youdas JW, Kotajarvi BJ, Padgett DJ, Kaufman KR. Partial weightbearing gait using conventional assistive devices. Arch Phys Med Rehabil. 2005;86(3):394-398.

10. Takanokura M. Optimal handgrip height of four-wheeled walker on various road conditions to reduce muscular load for elderly users with steady walking. J Biomech. 2010;43(5):843-848.

11. Melis EH, Torres-Moreno R, Barbeau H, Lemaire ED. Analysis of assisted-gait characteristics in persons with incomplete spinal cord injury. Spinal Cord. 1999;37(6):430-439.

12. Haubert LL, Gutierrez DD, Newsam CJ, Gronley JK, Mulroy SJ, Perry J. A comparison of shoulder joint forces during ambulation with crutches versus a walker in persons with incomplete spinal cord injury. Arch Phys Med Rehabil. 2006;87(1):63-70.

13. Kang Y, Na DL, Hahn S. A validity study on the Korean Mini-Mental State Examination (K-MMSE) in dementia patients. Journal of the Korean Neurological Association. 1997;15(2):300-308. 
14. O’Sullivan SB, Schmitz TJ. Assistive devices and gait patterns. In: Schnee M, editor. Physical Rehabilitation: Assessment and Treatment. Philadelphia, PA, USA: Davis; 2001.

15. Warren GL, Maher RM, Higbie EJ. Temporal patterns of plantar pressures and lower-leg muscle activity during walking: effect of speed. Gait Posture. 2004;19(1):91-100.

16. Hermens HJ, Freriks B, Merletti R, et al. European Recommendations for Surface Electromyography. Enschede, The Netherlands: Roessingh Research and Development; 1999.

17. Park S-Y, Yoo W-G. Comparison of exercises inducing maximum voluntary isometric contraction for the latissimus dorsi using surface electromyography. J Electromyogr Kinesiol. 2013;23(5):1106-1110.

18. Soderberg GL, Knutson LM. A guide for use and interpretation of kinesiologic electromyographic data. Phys Ther. 2000;80(5):485-498.

19. Sharkey NA, Marder RA. The rotator cuff opposes superior translation of the humeral head. Am J Sports Med. 1995;23(3):270-275.

20. Lee TQ, McMahon PJ. Shoulder biomechanics and muscle plasticity: implications in spinal cord injury. Clin Orthop Relat Res. 2002;403:S26-S36.

21. Mathiassen SE, Winkel J, Hägg GM. Normalization of surface EMG amplitude from the upper trapezius muscle in ergonomic studies - a review. J Electromyogr Kinesiol. 1995;5(4):197-226.
22. Naugle KM, Joyner J, Hass CJ, Janelle CM. Emotional influences on locomotor behavior. J Biomech. 2010;43(16):3099-3103.

23. Cacioppo JT, Priester JR, Berntson GG. Rudimentary determinants of attitudes. II. Arm flexion and extension have differential effects on attitudes. J Pers Soc Psychol. 1993;65(1):5-17.

24. Centerbar DB, Clore GL. Do approach-avoidance actions create attitudes? Psychol Sci. 2006;17(1):22-29.

25. Chen M, Bargh JA. Consequences of automatic evaluation: immediate behavioral predispositions to approach or avoid the stimulus. Pers Soc Psychol Bull. 1999;25(2):215-224.

26. Smidt GL, Mommens MA. System of reporting and comparing influence of ambulatory aids on gait. Phys Ther. 1980;60(5):551-558.

27. Liu HH, McGee M, Wang W, Persson M. Comparison of gait characteristics between older rolling walker users and older potential walker users. Arch Gerontol Geriatr. 2009;48(3):276-280.

28. Choi HJ, Ko CY, Kang S, Ryu J, Mun M, Jeon HS. Effects of balance ability and handgrip height on kinematics of the gait, torso, and pelvis in elderly women using a four-wheeled walker. Geriatr Gerontol Int. February 24, 2014. [Epub ahead of print].
Clinical Interventions in Aging

\section{Publish your work in this journal}

Clinical Interventions in Aging is an international, peer-reviewed journal focusing on evidence-based reports on the value or lack thereof of treatments intended to prevent or delay the onset of maladaptive correlates of aging in human beings. This journal is indexed on PubMed Central, MedLine,

\section{Dovepress}

CAS, Scopus and the Elsevier Bibliographic databases. The manuscript management system is completely online and includes a very quick and fair peer-review system, which is all easy to use. Visit http://www.dovepress. com/testimonials.php to read real quotes from published authors. 